\title{
Universidades y los dilemas de la calidad educativa en el contexto de la globalización
}

\section{Universities and the dilemmas of educational quality in the context of globalization}

\author{
Danny Guzmán Esquivel \\ Poder Judicial República de Costa Rica \\ dguzmane@poder-judicial.go.cr
}

\begin{abstract}
Resumen
El progresivo debilitamiento del poder estatal en Latinoamérica nos ha dirigido a tiempos de crisis en los cuales la constante presión de los poderes en el nivel global ha obligado a los gobiernos a plantearse situaciones tales como la apertura de los mercados y con esto el quebrantamiento de aquellas instituciones encargadas de velar por el bien social. Inmersos en un sistema capitalista avanzado que opaca y transforma la realidad de forma constante, vemos cómo el campo educativo no escapa a dichas circunstancias, lo que afecta no solo su estructura, sino también su razón de ser y su direccionamiento. En razón de esto, podríamos pensar en una educación que en vez de impulsar la integración del sujeto a un sistema busque la emancipación del ser humano, pero no en términos de industria y capital, sino que apunte a la emancipación del ser humano en el desarrollo de sus gustos y capacidades y lo lleve de la mano a nuevas alternativas.
\end{abstract}

Palabras claves: globalización, modelos neoliberales, calidad educativa, mercados, pedagogías críticas, pedagogías decoloniales 


\begin{abstract}
The progressive weakening of state in Latin America has led us to times of crisis, where the constant pressure from the powers at a global level have forced governments to consider situations such as the opening of markets and with this the breakdown of those institutions in charge to watch over the social goodness. Immersed in an advanced capitalist system that constantly obscures and transforms reality, we see how the educational field does not escape from such circumstances affecting not only its structure, but also its direction. Because of this, we could think of an education that instead of promoting the integration of the subject to a system, looks for the emancipation of the human being, but not in terms of industry and capital, but aim at the emancipation of the human being towards the development of their tastes and abilities, leading us to new alternatives.
\end{abstract}

Keywords: globalization, neoliberal models, educational quality, markets, critical pedagogies, decolonial pedagogies

\section{Introducción}

$\mathbf{E}$ s casi inevitable echar un vistazo atrás en nuestra historia, reflexionar sobre aquellos acontecimientos históricos que fueron la antesala de lo que hoy llamamos modernización; y es que a pesar de que este sea un concepto amplio y con un sinnúmero de variables por estudiar, lo cierto es que a partir de este nos hemos cruzado con dificultadas que todavía hoy nos cuesta sobrellevar, desde problemáticas ambientales hasta las de orden social en el que nuestro sistema económico ha llevado buena parte de la responsabilidad como transformador de las prácticas y modos de pensamiento en el quehacer humano.

Estas nuevas prácticas y modos de pensamiento han infiltrado prácticamente cualquier aspecto de la vida, han convertido nuestros dilemas existenciales en algo más que laberintos filosóficos sin salida y han guiado las posibles respuestas más bien hacia un asunto de obediencia a las reglas impuestas a modo de sobrevivencia, siendo la adhesión al sistema económico imperante la única razón de ser y existir, convirtiéndose estos últimos en el centro de nuestros problemas existenciales.

El tema de la educación no escapa en lo absoluto a estos dilemas; por el contrario, la educación superior, como se verá más adelante, es uno de los pilares con el que el sistema capitalista opera. Los centros educativos superiores (públicos, generalmente) se han proyectado tradicionalmente como aquellas trincheras de resistencias donde se fomenta el pensamiento crítico y se formulan propuestas para apaciguar el avance brutal y arrasador del capitalismo. Así, se cuestionará el papel que juegan estos en virtud de los viejos roles propiciadores de transformaciones, donde parece que en determinados momentos la lucha comienza a 
extinguirse al mismo tiempo que se cede progresivamente a las exigencias de los mercados neoliberales. Desde este punto es donde salta a la luz la discusión que mayormente ocupará este escrito que es la calidad educativa y el curso que esta ha venido tomando en los últimos años.

Si bien definir el término de calidad educativa resulta un tema de amplia envergadura, para efectos de este estudio se intentará partir desde aquellos elementos fundantes que van desde el surgimiento del mismo concepto en la educación hasta sus efectos y, por supuesto, sus transformaciones dentro de los diferentes contextos que vamos atravesando. A todo esto, en el apartado final se tocará la temática acerca de las posibles alternativas que tenemos para afrontar las problemáticas surgidas gracias a estas nuevas concepciones de calidad educativa desde nuestra región Latinoamericana y las propuestas que se han comenzado a formular.

\section{La globalización y los modelos neoliberales como generadores de nuevos modos de vida}

Los procesos de globalización y la implementación de políticas neoliberales han conllevado a una serie de cambios a lo largo y ancho del globo, no es posible pensar en estos procesos sin percatarnos de sus largos alcances en nuestras vidas afectando o modificando cualquier colectivo sin importar su posición geográfica, creencias o costumbres en general. Posterior a la caída de diversos grupos de izquierda que culminarían con la declive definitivo del bloque soviético (URRSS), una frase se popularizó a gran escala llevando dentro de sí, fuertes consecuencias que no hemos sido capaces de sobrellevar: el fin de la historia.

No es posible acabar con la historia, ni tan siquiera entender y analizar nuestros procesos de vida sin verlos en dicha retrospectiva, hay quien dice que "los tiempos pasados son siempre mejores", ya que siempre sentimos un anhelo de lo ya vivido, aunque evidentemente somos algo más, somos presente, futuro y pasado, nuestra existencia y nuestras acciones tal como lo presenta el materialismo dialéctico, están siempre interconectadas por nuestra historia, lo que fuimos, lo que somos y lo que seremos, relaciones, dinámicas y acontecimientos que se den dentro de estos planos de existencia. No obstante, el llamado fin de la historia intentaría condenarnos al olvido, a despojarnos de lo que somos, de lo que nos formó, la posibilidad de crear proyectos de sociedad alternativos a los impuestos, por lo contrario, el fin de la historia se nos implantaría como la supresión de nuestro pasado para imbuirnos completamente en una causa única, la causa del capital, donde la única posibilidad de sobrevivir se ha convertido en alinearse a las filas de la explotación del hombre por el hombre en un sistema donde la mala distribución de la riqueza es una de las principales fuentes de miseria para algunos, y para otros el boleto sin retorno para una vida de excesos y lujos desmedidos.

Como ya sabemos, buena parte de las herramientas del neoliberalismo consisten en ir quitándole presencia y potestad al aparato estatal, ya sea de forma acelerada o paulatina, el objetivo es claro, resaltar las supuestas ineficiencias del Estado y mostrar la privatización como la única vía posible para salir de una crisis que aparte 
de ser profunda y devastadora, es a su vez perpetua e inmortal. Desde nuestro contexto Latinoamericano nos resulta difícil pensar en un momento de nuestra historia en el que no hayamos estado imbuidos en algún tipo de crisis ya sea económica, política o social; siempre hemos sido territorios en los que se ha tenido que luchar constantemente, desde tiempos de la colonia para mantener nuestra autonomía, evitar el saqueo y la extracción por parte de los territorios que gozan de un poder económico y militar superior al nuestro.

"Pareciera que las representaciones
colonialistas sobre América Latina
fuesen generadas únicamente desde
los aparatos teórico-instrumentales de
los países colonialistas, lo cual dejaría
intocado el problema del modo en que
tales representaciones, en virtud de la
dinámica misma de la globalización,
son producidas también en Latinoamé-
rica. Ciertamente, las teorías poscolo-
niales tienen razón al mostrar que el
conocimiento científico de la moderni-
dad se encuentra directamente vincula-
do con la expansión del colonialismo"
(Gómez, 1998, p.13)

A este punto de la reflexión valdría la pena ponernos a pensar en aquello que nos determina, o al menos lo que pensamos que nos determina como latinoamericanos, y es aquí donde nos percatamos de que en cierta medida - sino es que en buena medida- somos una invención de los países desarrollados que al igual a como se designaron conceptos como "tercer mundo", o "subdesarrollo", la categorización de latinoamericanos sirve para colocarnos a priori en una posición periférica en relación al resto del mundo, Latinoamérica se convierte en una zona geográfica relevante únicamente cuando los recursos que allí se albergan fungen a favor de los intereses del capital extranjero para el desarrollo y crecimiento de sus corporaciones.

Cuando nos referimos a la entrada de un sistema neoliberal al mismo tiempo se está pensando en todos los escenarios que estos implican, empezando con la apuesta a la empresa privada como aquella capaz de cubrir todas las necesidades de subsistencia de los seres humanos, en vista de esto, la reducción del aparato estatal, nos ha venido dejando un escenario de incertidumbre y preocupación en la medida que los ataques sistemáticos a las instituciones públicas -incluyendo las educativas- se vuelven cada vez más frecuentes y con intencionalidades cada vez más focalizadas hacia la privatización más que en su fortalecimiento interno. La fuerte oleada de empresas extranjeras que ocupan los territorios latinoamericanos han generado un porvenir ausente de una promesa de prosperidad y esperanza a nuestras condiciones de desigualdad y sobre todo, al llamado subdesarrollo al que hemos sido relegados.

Sumando estos elementos a un claro avance hacia la consolidación de cada vez más monopolios, y el traslado de las iniciativas de bien social al ámbito privado, solo nos queda ahondar un tanto más acerca de dicho contexto en el plano educativo. Para ilustrar más este punto, basta echar un vistazo a las políticas impulsadas por el Banco Mundial en el año 1996 donde se ilustra la figura del Estado como regulador del aparato educativo, refiriéndose a este como un medio "que deja poco lugar para la flexibilidad que promueve un 
aprendizaje efectivo en el aula" citado en Muñoz (2006). Esta perspectiva expuesta en aquel entonces evoca un señalamiento que incita a mostrar el Estado como un obstáculo para formulación de cambios en los modelos de educación; dicho esto, no se puede negar el hecho de que el sistema educativo de nuestra región latinoamericana, continua arrastrando formas obsoletas en cuanto a la administración y gestión de la actividad educativa, sistema que no ha generado mayores esquemas de innovación en largo tiempo, al menos en el caso costarricense.

De acuerdo con lo anterior, nos acercamos a la temática de este escrito referida al contexto y las transformaciones de la educación superior en América Latina y como esto ha dado cabida a la creación de nociones de calidad distintas a las que se manejaban antes, dejando el mercado laboral como el núcleo de lo que se entiende como educación de calidad, en otras palabras, lo que se explicará más adelante es como el sentido de calidad educativa ese ha convertido en un producto prefabricado por parte de las exigencias del mercado laboral.

\section{¿La calidad educativa: ¿un concepto raptado por parte del mercado?}

De acuerdo con algunos estudiosos en el tema, entre ellos Vega (2012), la noción de calidad en el sistema educativo tiene su génesis en el ámbito empresarial, donde se sitúa el término "calidad" a la cualidad positiva de diversos objetos de uso común en la sociedad en relación con sus características de funcionamiento y duración. Posteriormente, en la década de los años 80, en tiempos en que el neoliberalismo salta a escena, el término calidad es transferido a los servicios públicos, incluida la educación. Desde allí, surgen diversos escritos relacionados con el tema, pero no es hasta 1983 que en los Estados Unidos se publica un documento en donde se establece una Comisión Nacional de Excelencia y, con esta, la fundación de la "calidad educativa" como una política de Estado, pero se mantiene casi intacta a esa noción de calidad ligada al mundo mercantil empresarial.

Como la calidad total proviene del mundo de la gerencia, a la educación se le atribuyen los mismos atributos que se le exigen a cualquier empresa: eficiencia, rendimiento, productividad incrementada a bajo costo, satisfacción de los clientes, competitividad, eficacia, innovación, rentabilidad, éxito y excelencia... Además, se supone que alcanzar todas esas metas debe ser una responsabilidad del centro educativo, de sus directivos y profesores, todos los cuales deben ofrecer una mercancía de calidad, en abierta competencia con todos los otros centros educativos, para satisfacer los gustos de los clientes. (Vega, 2012, p. 2-3)

Como puede observarse, el planteamiento del autor sugiere de forma enfática que uno de los principales problemas de la calidad educativa radica, en principio, en que aquello que se concibe como calidad educativa es una calidad diseñada y adecuada a la exigencia de los consumidores $y$, por ende, a los mercados educativos. Esta condición, que pareciera tener pocos puntos de salida, nos obliga constantemente a repetir los mismos patrones en los sistemas educativos una y otra vez, realizando pequeños ajustes pero siempre en beneficio de los sistemas económicos dominantes. 
La comprensión de lo que hoy es conocido como calidad educativa es producto de un proceso histórico promovido con fines específicos, en donde el auge acelerado de la ciencia y la tecnología en la era moderna dejaría, en consecuencia, la necesidad de formar cada vez más a individuos capaces de insertarse en los procesos de producción industrial, y como es de esperarse, la eficacia y la calidad educativas serían entendidas, manejadas y validadas tomando como parámetros las necesidades de la oferta y demanda de los mercados y su satisfacción en términos económicos.

De acuerdo con Vega (2012), la instauración de un principio de la calidad ya viene impregnada de un discurso ideológico basado en principios tecnocráticos que, como él mismo menciona, deja como resultado centros educativos análogos a los modos de operación de las empresas, lo que, como es de esperar, pone como objetivo la producción de capital económico. El sentido de la calidad educativa adquiere otros aspectos que han ido cobrando una importante relevancia en nuestros tiempos, aspectos que poco a poco se han ido generalizando, al punto de convertirse casi en una norma absoluta. Desde estas nociones, la estandarización de aquello que debe ser entendido como calidad educativa es visto a partir de certificaciones de calidad promovidas por agencias nacionales e internacionales, tomadas como objeto de crítica por unos, y por otros como una obligación necesaria que debe ser acatada a toda costa.

Siguiendo con el planteamiento de Vega (2012), la parametrización de la calidad educativa pasa por dos trampas intrínsecas que, como se menciona, han guiado y configurado las nociones de calidad educativa alrededor del mundo. La primera de estas trampas descansa sobre la idea de cómo el cliente entiende la cuestión de la calidad por medio del rendimiento académico de forma cuantificada, lo que da una cierta idea de objetividad, donde se le presenta un catálogo de universidades exitosas para escoger. Esto al mismo tiempo implica que la noción de calidad educativa quede sujeta, en buena medida, a los mecanismos publicitarios, reforzando así argumentaciones tales como que la cantidad de carreras acreditadas o la cantidad de estudiantes graduados anualmente son sinónimos de una educación de calidad. Al igual que cualquier mercancía, la calidad en estos términos es transferida a las necesidades del cliente, por lo que es importante señalar que un cliente de los mercados educativos universitarios puede ser tanto un estudiante quien vive directamente la experiencia en el centro educativo o, en su defecto, los padres y familiares o, en última instancia, quien financie los costos de la educación del estudiante, quienes si no logran estar conformes con el producto adquirido optarán por otro que se adecue a sus expectativas.

La segunda de las trampas que plantea el autor se fundamenta en la idea de que la calidad educativa tal y como es predicada en nuestra era actual, es canalizada en términos de competitividad, donde se intenta cuantificar aspectos como habilidades, destrezas y competencias, resultando así lo que se puede clasificar como un "darwinismo pedagógico", compuesto por ganadores y perdedores, depredadores y depredados en el campo de la educación. Es como sucede en las lógicas de libres mercados de 
los sistemas neoliberalistas, donde la "libre competencia" es la que va definiendo progresivamente quiénes adquieren un mejor posicionamiento, sumando a otros aspectos como el prestigio -ahora sí- la calidad de empresa o industria implicada en la batalla por la búsqueda del capital.

A partir de este tópico se pueden desprender inquietantes interrogantes acerca de qué se ha venido entendiendo por calidad educativa $\mathrm{y}$, sobre todo, si los parámetros estandarizados promovidos por las agencias acreditadoras son sinónimo de excelencia en la educación superior, tomando en cuenta que desde el año 2010 se decreta un artículo de ley en donde "el Estado y sus instituciones procurarán contratar personal de carreras oficialmente acreditadas", al tomar el Sistema Nacional de Acreditación de la Educación Superior (SINAES) como una de las plataformas para promover dichas iniciativas en la gestión de la calidad educativa en Costa Rica.

Aunque pudiera entenderse que esfuerzos como los expuestos anteriormente pueden ir guiados con buenas intenciones en pro del crecimiento y el mejoramiento de la calidad educativa en todas sus dimensiones, por otro lado llama poderosamente la atención cómo estos esfuerzos son asumidos como garantías incuestionables de buenas prácticas y buenos contenidos en cuanto a educación superior se refiere, a tal punto que esto termina por ser un atractivo para algunos centros educativos que lo utilizarán como plataforma publicitaria, atribuyendo la calidad de los servicios que se prestan a un equivalente de carreras acreditadas, y lo que llama un tanto más la atención, la cantidad de estudiantes graduados por año en estas universidades, como ya se mencionó.

Así como los buenos atributos de un fruto no pueden medirse por volumen de producción de un árbol, análogamente, la calidad educativa no puede medirse según de la cantidad de graduados que "produzca" un centro educativo, ya que esto nos remite a poner en cuestión las capacidades de responder a una demanda, mientras que la calidad educativa propiamente - a mi criterio- remite a la cantidad de herramientas teórico-metodológicas y la manera en que estas son culminadas en la praxis del quehacer profesional, que se esperaría desde una razón lógica se hallan en el proceso de formación universitaria.

Se considera esencial educar a la gente con habilidades cognitivas universales, en tanto que las funciones de socialización económica y política son decisivas para el bienestar de los individuos, comunidades y la sociedad entera. Los sistemas educativos desempeñan una función distributiva porque preparan a los individuos para sus funciones dentro de la división del trabajo; facilitan así una distribución eficiente del talento mediante la selección competitiva. (Torres, 1996, p.86)

Independientemente de si la formación de profesionales hoy en día va o no impregnada de lo que se puede denominar una calidad educativa real, es innegable que el valor de lo que puede significar "formarse profesionalmente" ha recaído en un sentido orientado especialmente a la inserción en el mercado laboral, reduciendo así las habilidades y los esfuerzos a las necesidades de este mercado. Como en algún 
momento lo mencionó Karl Marx, en los modos de vida capitalista los sujetos se encuentran tan ocupados de su subsistencia que no logran desarrollar sus verdaderas capacidades; de igual manera en el campo educativo, los estudiantes que de forma previa ven la educación como un instrumento de subsistencia más que un instrumento de formación en el que se requiere de un nivel de vocación y gusto, la elección de una profesión se convierte más en una elección de bien del mercado, para el mercado, orientado como se mencionó antes, en un medio de sobrevivencia que opera a partir de la inserción y la adopción de las normas que exigen las pautas de un mundo mercantil.

Como se explicó desde un inicio, el sentido de calidad educativa fue promovido en un momento en el que el auge industrial comenzaba a arraigarse aún más en la vida social. Bajo esta noción vemos cómo aspectos propios de la industrialización como la rapidez, la eficacia y la eficiencia son trasladados al campo educativo donde sujetos(as) de estratos sociales diferentes, con aspiraciones distintas, y todavía más importante, habilidades distintas, son puestos en un espacio que si bien no denota a simple vista un campo de batalla, simbólicamente funciona como tal y reproduce, a la larga, principios de competencia que posteriormente serán recompensados empezando por los maestros hasta el mismo sistema educativo que es a final de cuentas se encargará de situar a cada estudiante en una posición mejor o peor de acuerdo con la tabla de calificaciones obtenidas; pareciera que en buena parte de los aparatos educativos del mundo, este es el único medio por el que se pueden "medir" las capacidades de una población estudiantil.

Entonces, por un lado no resulta extra-
ño que, en especial en los últimos años,
la obsesión por la calidad haya des-
embocado en una retórica que la sitúa
como un fin en sí misma, lo que lleva
a perder de vista las múltiples dimen-
siones implicadas en una educación de
calidad y a desconocer la atención inte-
gral y armónica de las condiciones que
ella demanda. (Cruz y otros autores,
2009, p.170)

La definición de calidad educativa encierra en sí misma un complejo entramado de concepciones, muchas de las cuales tenderán a caer en el relativismo; otras tenderán a crear parámetros de medición a partir de múltiples variables como las tendencias utilizadas en las acreditaciones de las carreras universitarias. Separadamente de buscar una definición absoluta o relativista para delimitar el término calidad educativa, es importante, como lo muestra el autor citado anteriormente, no descuidar aspectos como la atención integral y armónica cuando se intenta establecer un concepto pleno al referirnos a la calidad educativa, ya que como este sostiene, la idea de calidad educativa ha recaído sobre aspectos que no necesariamente contribuyen a la construcción de un sentido integral de calidad, sumando algunos elementos como el marketing de algunas universidades que ven la práctica educativa dentro de un eslabón más para la generación de capital y no como espacios de formación de quienes se supone serán los que tomen las riendas de una nación. 


\section{Respuestas al modelo educativo imperante}

Ya expuestos algunos de los aspectos medulares que ayudaron a confeccionar este escrito desde una perspectiva crítica y sobre todo reflexiva, es necesario pensar en las posibles alternativas que tenemos ante la corriente que comienza a arrastrar nuestro sistema educativo superior que, como se puede evidenciar, nos dirige hacia una concepción de educación dominada por la homogenización del conocimiento a fin de que aquellos sectores que poseen los medios de producción alrededor del planeta puedan contar con mano de obra al mejor precio posible para que operen las grandes corporaciones y todo lo que las rodean, al mismo tiempo que el mantenimiento del poder estatal se ve cada vez más como la opción menos viable y se formula, por el contrario, estrategias para su debilitamiento.

Por otro lado, con el paso de la modernidad y los procesos expansionistas de las grandes potencias, de manera casi inmediata nos percatamos de las imposiciones gestadas desde estas élites en donde el avance acelerado de estos sistemas ha calado fuertemente en nuestros inconscientes, tal y como lo plantean las teorías asociadas a la colonialidad del poder, la colonialidad del ser o la colonalidad del saber.

La disputa en el campo del saber implica, de forma implícita, choques en los que se pone en juego algo más que una competencia acerca de quién se encuentra más o menos cerca de alcanzar un conocimiento más válido, o quién es el primero en llegar a este; las disputas libradas en el campo del conocimiento en el contexto de la modernidad comprenden en sí mismas un armazón complejo en el que ciertos grupos dominantes, los poseedores del conocimiento hegemónico, legitimado y empleado como una forma de conocer, han opacado otros muchos saberes, desplegando, sobre el terreno del saber, lógicas de pensamiento de corte epistemicidas que han minado las posibilidades de redireccionar $\mathrm{o}$, en su defecto, reconstruir el quehacer de la ciencia desde nuestras regiones, siendo la razón eurocéntrica la que sigue contando con la última palabra, mientras se comienzan a promulgar esfuerzos para hacer frente a dichas perspectivas como las pedagogías críticas o las pedagogías decoloniales.

Dadas las circunstancias actuales en las que los diferentes círculos académicos se han prestado como espacios de intercambio de ideas y ejes de acción para el cambio social, es innegable que estos mismos espacios han funcionado también como escenarios donde se han edificado "templos sagrados" que han contribuido a la tipificación de académicos como maestros absolutistas poseedores de todo conocimiento, portadores de verdades incuestionables, siendo esta realidad no muy ajena a los pensadores de corte latinoamericanista, como bien lo dejan en evidencia autoras como Cusicanqui (2010), quien denomina dichos círculos académicos como un legítimo canon académico que no ha hecho más que agudizar la jerarquización de saberes en el campo de la educación, justamente porque se tiende a centralizar el quehacer académico en el incremento del capital simbólico y cultural bajo la bandera del conocimiento de 
los pueblos indígenas y afrodescendientes más no en un acercamiento real con estos.

Tomando en cuenta lo anterior, se entiende como principio fundamental que las pedagogías decoloniales llevan consigo una fuerte adhesión a la interculturalidad, pensando la realidad como un todo en el que interactúan diferentes grupos sociales en los que sus visiones de mundo si bien pueden diferir unas de otras, también se considera la posibilidad de generar espacios de convergencia entre estos como la respuesta para mediar escenarios en los que se puedan superar los constructos epistemológicos perpetuados desde las academias occidentalizadas.

Desde aquí es donde las pedagogías decoloniales pretenden crear espacios de transformación donde en conjunto con la interculturalidad, se pueda no solo reconocer las otredades, sino también construir proyectos de sociedades en los que se reconozcan las diversificaciones de saberes $\mathrm{y}$, con esto, la generación de conocimiento desde fuera de las imposiciones tradicionales. Pareciera que nos encontramos sumidos en una hegemonía tecnocrática y mercantil que ha reclamado como propia buena parte del quehacer académico, hecho que puede verse mayormente reflejado en el campo de la educación privada donde aquellas carreras con una mayor demanda son las que terminan ocupando lugares privilegiados.

Dentro de este panorama, pueden verse algunos esfuerzos que a pesar de no poder catalogarse como ejemplos de pedagogías críticas desde un sentido estricto, de alguna manera dentro de sus programas reflejan un esfuerzo por acercar su pensamiento y sus valores en tal dirección. Un ejemplo puede manifestarse en programas como el de Estudios Latinoamericanos de la Universidad Nacional de Costa Rica, donde se pone a disposición de los estudiantes conocimientos relacionados con las pedagogías críticas que buscan propiciar espacios en los que se discuta el quehacer de la educación desde un perspectiva en la que se vea el salón de clase como un lugar de construcción mutua entre profesor y estudiantes, siempre viendo la educación como una herramienta para fomentar el cambio.

La muerte de los sueños y de la utopía, una consecuencia derivada de la muerte de la Historia, supone la inmovilización de la Historia reduciendo el futuro a la permanencia del presente. El presente victorioso del neoliberalismo es el futuro al que nos adaptaremos... No basta con reconocer que el sistema actual no nos incluye a todos. Precisamente porque lo reconocemos, es necesario luchar contra esa evidencia y no adoptar la postura fatalista adoptada por ese mismo sistema... (Freire, 2012, p.159-160)

Parte del camino por recorrer para propiciar los cambios necesarios también implica una variación en las mediaciones pedagógicas que se emplean en las aulas, atenuando de esta manera alternativas que nos permitan deconstruir las viejas doctrinas eurocentristas que nos han gobernado por años; dicho de otra manera, la apuesta por nuevos modelos educativos y la regeneración del concepto de calidad educativa vendrían por la generación de espacios que nos permitan desaprender 
las viejas doctrinas, comenzando así con la edificación de nuevos paradigmas en la educación superior, espacios que, como ya se ha expresado, busquen la superación de las visiones de los mercados educativos que tienen como fin la comercialización de la enseñanza como lo hace cualquier empresa encargada de prestar servicios que tienen como último fin la reproducción de relaciones entre vendedores y consumidores. Superado esto, quizá podamos encontrarnos un paso más cerca no solo de una resignificación de la educación, sino que también podríamos empezar a construir los primeros escalones que nos guíen hacia nuevas experiencias sociales capaces de transformar la realidad social.

Y es precisamente desde ese punto donde se puede comenzar a trabajar, desde nuestro propio entendimiento de la realidad social y, sobre todo de nuestro posicionamiento en contraste con lo que somos. Es posible dar cuenta de que buena parte de la aceptación de nuestras condiciones de subordinación son validadas en pro de la supremacía blanca europea donde la tradición colonial cargada de una supuesta legitimidad biologicista-religiosa termina por otorgar el derecho de explotación a las poblaciones indígenas y a las poblaciones negras que ya vendrían como población esclavizada por los conquistadores. Bajo esas condiciones es como sea crea el mito del "blanco americano" y lo que se conocería con el pasar de los años como criollos, con el fin de invisibilizar nuestros verdaderos orígenes que no son más que la confluencia de diversas culturas; justo aquí es donde puede evidenciarse uno de los principales retos de las pedagogías críticas (o en su defecto, las pedagogías decoloniales): la tarea de autovisibilizar nuestros orígenes.

No importa si las propuestas puedan ir orientadas desde las pedagogías críticas o las pedagogías decoloniales; más allá de eso, pareciera que es más importante poner en evidencia que en el campo educativo no podemos seguir adheridos a viejas doctrinas y no en razón de su antigüedad únicamente, sino haciendo conciencia de que estás formas de "crear" y de "formar" nos están arrastrando a un abismo, el abismo de la dominación extranjera, el abismo de la imposición y la deshumanización.

\section{Conclusiones}

La discusión acerca de la calidad educativa nos remite de forma inevitable a un entramado complejo acompañado de un arrastre sociohistórico que ha sido objeto de amplias discusiones que, a fin de cuentas, despliega una serie de variantes que involucran problemas de orden económico y político donde, como se pudo evidenciar, se juega algo más que un tema de "buena calidad educativa", o de mejoras en las herramientas pedagógicas que se emplean $\mathrm{y}$, como se pudo ver a lo largo de este estudio, los juegos de intereses de poder se encuentran de forma implícita dentro de los "planes de mejora" en la educación superior.

En múltiples ocasiones se oye hablar de una necesidad de desarrollar reformas educativas, y desde esta necesidad se comienzan a discutir temas como el recorte presupuestario a la educación superior, la creación de nuevos parámetros de evaluación de los programas y así cientos de propuestas que parecieran ir en pro de 
las mejoras que se requieren para una educación de calidad. También es importante destacar que ante la crisis laboral se da inicio a una "puesta de etiquetas" orientada a la clasificación de las carreras universitarias, generando así lo que puede denotarse como órdenes jerárquicos entre las distintas profesiones a partir de su cotización en los mercados, siendo los límites entre un médico y un filósofo suficientes para la colocación de uno y otro en posiciones diametralmente opuestas, y no necesariamente por sus conocimientos, sino por su poder adquisitivo.

Calidad educativa es un término ambiguo, claro está, y su definición puede incluir variables casi interminables; sin embargo, existe una necesidad que nos convoca a repensar lo que hemos estado entendiendo por calidad educativa y lo que estas concepciones están incrustando en los imaginarios sociales, así como lo que debería entenderse como desarrollo social, ya que la satisfacción personal, la vocación y el gusto por lo que se hace han venido remplazándose por una necesidad de sobrevivencia al sistema económico y la búsqueda de un estatus social y económico que no podrá alcanzarse por medio de cualquier profesión a menos que se cuente con los recursos materiales de forma previa y en demasía. Hay una necesidad de cambio, pero este cambio deberá hacerse desde las bases que componen nuestra existencia y el sentido que le damos a esta, ya que validar y legitimar nuestras condiciones ya no son una opción; como seres humanos necesitamos algo más que sobrevivir a un mercado.

El fenómeno de la privatización y los cambios estructurales económicos y políticos experimentados desde los años 80, nos han dejado como resultado una clara reorganización de nuestras dinámicas sociales que, como es de esperar, llegarían a impregnar el sistema educativo y del sistema de educación superior que hasta el día de hoy se ha convertido en un campo en disputa por su valor y peso dentro de la formación de profesionales con pensamiento crítico, quienes a menudo suelen incomodar a ciertas élites.

En lo que respecta Costa Rica, el Estado de la Educación publicado en el año 2015 nos muestra que las áreas de Ciencias Sociales, Ciencias Económicas y Educación representan la mayor parte de la demanda educativa ( $58 \%$ en total) que; dentro de estas, son las universidades privadas las que cuentan con una mayor oferta representa un $70,7 \%$ del total, y las universidades públicas un $46,8 \%$. Presentado este panorama, vemos cómo de manera evidente las universidades privadas desde su periodo de mayor crecimiento (desde los años 90) han logrado tomar un lugar de relevancia en la formación de profesionales. Puesto lo anterior en contexto, parece importante entender la realidad desde distintas ópticas que nos permitan ubicar nuestra situación global, las necesidades del mercado y cómo los mercados educativos interactúan, penetran y transforman el sentido propio de la educación superior, ya que estos aspectos guardan relación directa con lo que se puede entender como calidad educativa. Es precisamente desde aquí donde vemos uno de los principales retos en la educación universitaria, que ante un arrasamiento casi imparable de los modelos imperantes, debe darse a la tarea de habilitar espacios de discusión en 
los centros estatales, pero aún más en los centros de estudios superiores privados ya que esto permitiría empezar a retorcer los adoctrinamientos de orden mercantil que nos gobiernan. Pedagogías críticas en una educación homogenizadora es de forma casi contundente la llave para propiciar cambios profundos en la educación superior; es la forma, quizá, de construir profesiones con alma.

\section{Referencias}

Castro, S. (1998). Latinoamericanismo, modernidad, globalización: Prolegómenos a una crítica poscolonial de la razón. México: Editorial Porrúa.

Cruz, O. y otros (2009). ¿Calidad de la educación o educación de calidad? Una preocupación más allá del mercado. Revista Iberoamericana de Educación. España.

Cusicanqui, S. (2010). Ch'ixinakax utxiwa: una reflexión sobre prácticas $y$ discursos descolonizadores. Buenos Aires: Ediciones Tinta Limón.
Freire, P. (2012). Pedagogía de la indignación: Cartas pedagógicas a un mundo revuelto. Buenos Aires: Siglo Veintiuno Editores.

Muñoz, V. (2006). El oro por las cuentas. Miradas a la mercantilización de la educación. San José: Ed. Luna Híbrida y Centro de Investigación y Docencia en Educación, Universidad Nacional.

Molina, I. (2016). La educación en Costa Rica de la época colonial al presente. San José: Editoriales Universitarias Públicas Costarricenses.

Torres, C. (1996). Las secretas aventuras del orden. Estado y Educación. Buenos Aires: Miño y Dávila Editores

Vega, R. (2012). La calidad educativa, una noción neoliberal propia del darwinismo Pedagógico. Revista Rebelión. España. 
